\title{
PIRD: Pan immune repertoire database
}

Wei Zhang ${ }^{1,2, \dagger}$, Longlong Wang ${ }^{1,2,4, \dagger}$, Ke Liu ${ }^{1,2, \dagger}$, Xiaofeng Wei ${ }^{1,2, \dagger}$, Kai Yang ${ }^{1,2}$, Wensi Du ${ }^{1,2}$, Shiyu Wang ${ }^{1,2,4}$, Nannan Guo ${ }^{1,2}$, Chuanchuan Ma ${ }^{1,2}$, Lihua Luo ${ }^{1,2}$, Jinghua Wu ${ }^{1,2}$, Liya Lin ${ }^{1,2}$, Fan Yang ${ }^{1,2}$, Fei Gao ${ }^{1,2}$, Xie Wang ${ }^{1,2}$, Tao $\mathrm{Li}^{1,2}$, Ruifang Zhang ${ }^{1,2}$, Nitin K. Saksena ${ }^{1,2}$, Huanming Yang ${ }^{1,3}$, Jian Wang $^{1,3}$, Lin Fang ${ }^{1,5}$, Yong Hou ${ }^{1,2}$, Xun Xu ${ }^{1,2}$, Xiao Liu ${ }^{1,2, *}$

${ }^{1}$ BGI-Shenzhen, Shenzhen, 518083, China

${ }^{2}$ China National GeneBank, BGI-Shenzhen, Shenzhen 518120, China

3James D. Watson Institute of Genome Sciences, Hangzhou 310058, China

${ }^{4}$ BGI-Education Center, University of Chinese Academy of Sciences, Shenzhen, China.

${ }^{5}$ Department of Biology, University of Copenhagen, Copenhagen, Denmark

* To whom correspondence should be addressed. Tel: 086-13438700710; Fax:; Email: liuxiao@genomics.cn

tThese authors contributed equally to the paper as first authors. 


\section{ABSTRACT}

Motivation: $T$ and $B$ cell receptors (TCRs and BCRs) play a pivotal role in the adaptive immune system by recognizing an enormous variety of external and internal antigens. Understanding these receptors is critical for exploring the process of immunoreaction and exploiting potential applications in immunotherapy and antibody drug design. Although a large number of samples have had their TCR and BCR repertoires sequenced using high-throughput sequencing in recent years, very few databases have been constructed to store these kinds of data. To resolve this issue, we developed a database.

Results: We developed a database, the Pan Immune Repertoire Database (PIRD), located in China National GeneBank (CNGBdb), to collect and store annotated TCR and BCR sequencing data, including from Homo sapiens and other species. In addition to data storage, PIRD also provides functions of data visualisation and interactive online analysis. Additionally, a manually curated database of TCRs and BCRs targeting known antigens (TBAdb) was also deposited in PIRD.

Availability and Implementation: PIRD can be freely accessed at https://db.cngb.org/pird.

\section{BACKGROUND}

T cells and B cells, as the two pillars of the adaptive immune system, have a crucial role in health and disease. T cell receptors (TCRs) and B cell receptors (BCRs) undergo clonal expansion or antibody affinity maturation to react against antigens. The majority ( 95\%) of T cells express TCRs composed of $\alpha$ and $\beta$ chains ( $\alpha \beta$ T cells), while the rest of the TCRs are composed of gamma and delta chains ( $\gamma \delta$ T cells). Similarly, the BCRs expressed on the B cell surface consist of heavy and light chains. During the development of $\mathrm{T}$ and $\mathrm{B}$ lymphocytes, recombination of variable $(\mathrm{V})$, diversity $(\mathrm{D})$ and joining $(\mathrm{J})$ gene segments (a range of $\mathrm{V}, \mathrm{D}$ and $\mathrm{J}$ gene segments ranked on chromosome), nucleotide deletion at the end of gene segments and nucleotide insertion during the V-D or D-J junctions (V-J junction for $\alpha, \gamma$ and light chains) cause a highly diverse array of TCR and BCR repertoires (Liu and $\mathrm{Wu}, 2018$ ). Theoretically, more than $10^{18}$ unique TCRs and more than $10^{13}$ unique BCRs have been predicted for human beings (Georgiou, et al., 2014; Venturi, et 
al., 2008). Therefore, the extremely large diversity of receptors promotes the ability of the immune system to maintain homeostasis in health and disease.

With the advances in high-throughput sequencing platforms, there has been a rapid accumulation of TCR and BCR repertoire sequencing data in the past decade. Specifically, since several influential and seminal studies published in 2009(Boyd, et al., 2009; Robins, et al., 2009; Weinstein, et al., 2009), an increasing number of studies and applications have occurred in subsequent years, which included (i) investigating the immune microenvironment in tumours to assist in therapy by searching for tumour-infiltrating $T$ cells targeting neo-antigens (Reuben, et al., 2017; Riaz, et al., 2017; Wang, et al., 2017; Zhang, et al., 2017; Zheng, et al., 2017); (ii) detecting minimal residual disease and monitoring immune reconstitution after treatment and transplant( $\mathrm{Wu}$, et al., 2012; $\mathrm{Wu}$, et al., 2016); (iii) exploring immune characteristics in autoimmune and infectious diseases and evaluating the effects of vaccines(Gomez-Tourino, et al., 2017; Jackson, et al., 2014; Jiang, et al., 2013; Parameswaran, et al., 2013; Smithey, et al., 2018; Wang, et al., 2018); (iv) identifying disease-associated clones(Dash, et al., 2017; Emerson, et al., 2017; Glanville, et al., 2017; Huang, et al., 2019); and (v) screening the production of monoclonal antibodies targeting specific antigens and human immunodeficiency virus (HIV) by broadly neutralizing antibody(Cheung, et al., 2012; Jardine, et al., 2016).

TCR and BCR sequencing data are valuable resources; thus, a special database to collect and store these sequencing data is highly needed. Recently, the Adaptive Immune Receptor Repertoire (AIRR) Community released a data standard for sharing and developed the platform iReceptor for querying immune repertoire data(Corrie, et al., 2018; Rubelt, et al., 2017); however, iReceptor only provides a primary search function. Here, we have developed a multifunctional database, PIRD, that stores the TCR and BCR sequence information and provides the functions of data statistics, visualisation and interactive online analysis. Additionally, a database of disease- and antigenassociated sequences, TBAdb, was also deposited in PIRD. PIRD provides the opportunity for other investigators to reuse the data more easily and to compare different aspects of the data. The database may aid in the development of disease treatment, drug research and vaccine designs.

\section{MATERIAL AND METHODS}

\section{Data Sources}

To upload the data to PIRD, three datasets are required, which include the project information, sample information and annotated TCR or BCR repertoire sequences. The TCR or BCR repertoire can be captured for a single chain by multiple PCR(Liu, et al., 2016; Robins, et al., 2009), rapid amplification of cDNA ends (5'-RACE)(Warren, et al., 2011), unique molecular identifiers (UMI) (Khan, et al., 2016; Turchaninova, et al., 2016; Vollmers, et al., 2013) and other methods. To obtain 
paired chains of receptors, such as TCR $\alpha$ and $\beta$ chains and BCR heavy and light chains, singlecell RNA sequencing technology(Zheng, et al., 2017) and other developed methods(DeKosky, et al., 2013; DeKosky, et al., 2015; Howie, et al., 2015) can be used. These repertoires are then sequenced by high-throughput sequencers, such as Illumina(Zhang, et al., 2017), BGISEQ(Huang, et al., 2017) and 454(Wang, et al., 2015). The sequences can be processed by commonly used tools, such as MiXCR(Bolotin, et al., 2015), MiTCR(Bolotin, et al., 2013), IMonitor(Zhang, et al., 2015), IgBLAST(Ye, et al., 2013), and Change-O(Gupta, et al., 2015), to generate the annotated information. Both gDNA and RNA of lymphocytes derived from peripheral blood and specific tissues can be used to generate the repertoire. Generally, PIRD only collects data from research studies that have been previously published or that are planned to be published in the future. TBAdb, a relatively independent database deposited in PIRD, contains the antigen and diseaseassociated TCR and BCR sequences that were derived from previously published literature.

\section{Database Implementation}

The implementation of the web application and database was performed in Django, a high-level Python Web framework. The framework of the search engine and data flows of PIRD was performed using Elasticsearch (a distributed data and real-time search engine), which provides association analysis and real-time and seamless retrieval for all data into the database. PIRD depends on the nodes built by MongoDB clusters and Elasticsearch clusters that standardize and integrate data. Additionally, the development of online analysis and the visualisation platform were based on the MongoDB framework.

\section{RESULTS}

\section{A Brief Introduction and Data Types In PIRD}

PIRD mainly includes five basic datasets, including project information, sample information, raw sequencing data, annotated TCR or BCR repertoires, and TBAdb (Fig. 1). First, the project information contains the description of the project, data processing standards, primers used, sample size, and types of receptors, species, individuals, etc. Importantly, one individual may have multiple samples included in the database; this information is listed here. Second, the sample information is made up of two types of data. One type is the data associated with sample collection, processing and phenotype, such as cell origin, receptor type, sequencing platform, gender, age and race. The other type is the overall statistics of TCR or BCR repertoires, which include total sequence number, unique complementarity-determining region 3 (CDR3) number, diversity index (Shannon index), top 10 clones and frequencies, CDR3 length distribution, and V/J/V-J gene usage. Third, raw sequencing data, deposited in the Nucleotide Sequence Archive (CNSA, https://db.cngb.org/cnsa/), which is a newly developed database to store different kinds of raw sequencing data, can be viewed by clicking the weblink provided in PIRD. Fourth, the dataset of 
annotated TCR or BCR repertoires is the core resource. The annotation is displayed by each unique sequence, including the information of CDRs and framework region (FR) fragments, VDJ assignment, deletion/insertion nucleotides, paired chains, sequence amount, and mapping information. Last, there is a special independent database, TBAdb, deposited in PIRD.

\section{Summary of Data Storage}

The data in PIRD are deposited independently by project (Fig. 2B). Thus, the project is the gateway for users to view all data and other information associated with the project (Fig. 2A). Specifically, project information, sample information and raw data can be viewed by clicking 'ProjectID'. To visit the annotated TCR or BCR repertoire, users can click the 'SampleID' shown in the statistics or information pages of 'sample'. For each sequence, 27 items are used to annotate it by immune repertoire format (IRF-V1.0, https://db.cngb.org/pird/tools/), including VDJ gene assignment, CDR and FR identification, deletion and insertion nucleotides, and mapping information (Fig. 2A). As of July 2019, there are 3657 samples in PIRD, including healthy samples, cancers, autoimmune disease and vaccines, with 877 systemic lupus erythematosus (SLE) samples, 439 healthy samples and 169 leukaemia samples (Fig. 2C). The total number of sequences in PIRD reached 11,395 million $(\mathrm{m})$, and the phenotypes with the top 3 abundant sequences were $2539 \mathrm{~m}$ in $\mathrm{IgA}$ nephropathy project, $1924 \mathrm{~m}$ in minimal residual disease (MRD) project and $1920 \mathrm{~m}$ in healthy samples (Fig. 2C). Approximately $76.65 \%$ of samples in PIRD are TCR $\beta$ chain, while others are heavy chain (Fig. 2C). The data in PIRD will be updated over time. In the future, the data will gradually increase as more academic papers are published.

\section{Functions of the Database}

PIRD provides data storage, data statistics and visualisation, interactive online analysis and visualisation, search, and uploading and downloading of data (Fig. 1). First, as the fundamental function of PIRD, data storage includes all information of five basic datasets. Second, for each sample, statistics such as CDR3 diversity, CDR3 length distribution, and V/J/V-J pairing usage are calculated from the annotated TCR or BCR repertoires (Fig. 1). To facilitate visualisation by users, PIRD provides corresponding figures with a single click of the button. An example shows one sample's V-J gene pairing and CDR3 length distribution (Fig. 3A). Additionally, we provide figures for clinical and other data pertinent to the projects, such as the age distribution and CDR3 diversity distribution (Fig. 3B). The third function of the database is the online interactive analysis. We offer a simple and user-friendly platform for users, even those who lack bioinformatics skills, to compare and visualise datasets. For the search function, PIRD provides five types of search modules, including by project, sample, clone, TBAdb and basic local alignment (BLAST) (Fig. 3C). The first three types contain multiple items for users to choose to search for their interested data. The fourth type only searches the data in TRAdb. The last type is a fuzzy search that finds regions of local similarity between sequences using BLAST tool(Altschul, et al., 1990; Ye, et al., 2006) (Fig. 3C). 


\section{Interactive Online Analysis With PIRD}

To facilitate direct analysis of data in PIRD, we developed a platform for interactive online analysis. Users can upload their own data or select only specific data in PIRD to create a datasheet. Some analyses, such as comparison of different group features, can be performed, and the corresponding figures, including histogram plot, line plot and box plot, will be generated, which can be performed by dragging the buttons on the left of the website page (Fig. 4A). We used the data of the patient (I190114010005) from a breast cancer-associated project (P19011401) in PIRD as an example and found inconsistent CDR3 length and TCR V $\beta$ gene usage distributions among tumour, adjacent normal tissue and lymph node (Fig. 4B). In addition, we compared the data from multiple projects in PIRD, including those on colon cancer (P18081201), breast cancer (P19011401), healthy people (P18080801) and SLE samples (P18081001). We found that the $\mathrm{BCR}$ repertoire diversity of intestinal segment $\mathrm{C} 1$ is lower than that of other segments, and the diversity between tumour and adjacent normal tissues is significantly different (Fig. 4C, left panel). Regarding the TCR repertoire, we found that the diversity of SLE is lower than that of the healthy group, and the diversities among different types of samples in breast cancer patients are inconsistent (Fig. 4C, right panel). Overall, the greatest advantage of this analysis platform is that multiple types of diseases or projects deposited in the PIRD can be easily compared.

\section{Superiorities of PIRD}

To date, several repositories have been established to store TCR and BCR repertoire sequences, including ImmuneDB(Rosenfeld, et al., 2017; Rosenfeld, et al., 2018), VDJServer(Christley, et al., 2018) and iReceptor(Corrie, et al., 2018). ImmuneDB and VDJServer mostly focus on online platforms for users to analyse TCR and BCR repertoire sequences. Although users can store the analysed results and share the data in the website of VDJServer, currently, the shared data is very limited . iReceptor is the scientific gateway of the database generated by the AIRR community. Several working groups in the AIRR community, including a common repository group and a minimal standards group, have provided a general standard for sharing the data(Rubelt, et al., 2017). PIRD provides information and data processing pipelines that can be adapted to AIRR standards(Rubelt, et al., 2017). Currently, PIRD provides more functions than iReceptor. First, PIRD provides an interactive online analysis platform, where users can perform some comparison analyses. Second, PIRD offers more statistics and visualisations, such as overall diversities, CDR3 length distribution and V/J gene usage for each sample (ProjectID->Sample->Statistics). Third, for each sequence, the information on deletion and insertion nucleotides, detailed positions of fragments (CDRs and FRs), and paired chains is also included. Fourth, PIRD provides a more powerful search function, including the fuzzy search by BLAST tools. Last, as of July 2019, PIRD has more TCR and BCR sequences than iReceptor (3657 vs. 879 samples, $11,395 \mathrm{~m}$ vs. $1,300 \mathrm{~m}$ sequences), which is mostly due to a collaboration with the Pan-Immunome Initiative(Xiao Liu, 2018) to release its data before publication. Both PIRD and iReceptor have been trying to provide 
an application programming interface (API) for users to easily access data from both databases in the near future.

\section{TBAdb: a manually curated Database of T and B cell receptors targeting known Antigens}

Antigen-specific TCRs or BCRs are crucial resources for disease assessment, therapy and vaccine development. To collect previously reported disease and antigen-associated sequences, we read hundreds of published studies and created a knowledge database of TCR and BCR sequences with relevant information. The information collected for each sequence was manually curated and critically checked. The fields include the disease name, antigen, CDR3 sequence, V/J usage, HLA type, experimental method, grade, publication details, etc. Importantly, to evaluate the quality and reliability of antigen or disease specificity, we provide a grade for each sequence according to the identification methods reported in the literature. The criteria for grades are described in the database website (https://db.cngb.org/pird/tools/). TRAdb contains three parts, including TCR $\alpha \beta$, TCR $\gamma \delta$ and BCR. It currently includes 52,287 sequences, with 71 diseases. The diseases are divided into five categories, including autoimmunity, cancer, pathogen, allergy and other, like the categories of database McPAS-TCR(Tickotsky, et al., 2017). For the first three categories, each one contains more than 14 diseases (Fig. 5A). In TBAdb, the pathogen category accounts for most of the sequences, which is $89.51 \%$ in total (Fig. $5 \mathrm{~B}$ ). We also found that most of the sequences in the pathogen category are more reliable (grade $>4$, Fig. 5D). This could be because the pathogenic diseases have been widely researched, and the TCR or BCR sequences targeting pathogens are relatively easy to identify. The category with the second largest proportion is autoimmune (Fig. 5B), but more than half of the sequences are shown with a lower grade (Fig. 5D). Although the other category contains 13 diseases, it accounts for only $0.96 \%$ of sequences, and most of them are less reliable (Fig. 5D). For TCR $\alpha \beta$ sequences, TBAdb contains more data than the VDJdb(Shugay, et al., 2018) and McPAS-TCR(Tickotsky, et al., 2017) databases; however, we found that $9.7 \%$ and $5.1 \%$ of sequences overlap with the two databases, and $2.3 \%$ of sequences are shared among the three databases (Fig. 5C). Therefore, TBAdb is a good supplement for TCR $\alpha \beta$ sequences. In addition, TBAdb also includes the data of TCR $\gamma \delta$ and BCR.

\section{DISCUSSION}

Here, we described the PIRD database, a unified repository to store immune repertoire data. The lack of annotated repositories required for immune repertoire sequence storage prompted the creation of PIRD. PIRD is located at CNGBdb (https://db.cngb.org/, Shenzhen, China), which is a newly developed integrative and interactive database. The data in PIRD can be found according to the similarity of sequences from the search page of the CNGBdb. In summary, this multifunctional database provides several benefits to users. First, identifying the disease- or antigen-specific clones is the main purpose of some studies; however, the high diversity of the 
immune repertoire and very low overlapping rate between individuals(Glanville, et al., 2011) require large-scale samples to evaluate the specificity and sensitivity of the associated clones. Therefore, a wide range of samples in PIRD could provide the function of evaluating clones' reliability. Second, the bioinformatic methods for immune repertoire data are still in their infancy. Some clustering methods of TCR sequences targeting the same antigen have been recently reported(Dash, et al., 2017; Glanville, et al., 2017), and more machine learning methods will be developed in the future due to the increase in the sequencing data. Thus, the data in PIRD can aid in the development and assessment of new methods by providing training and testing data. Additionally, the sequences in PIRD can also be reused for data mining by the new methods. Third, the online analysis function of the database offers a valuable platform for users to analyse the interested data with a simple interactive interface.

In the future, the online analysis platform in PIRD will be further optimized and developed, including a more friendly interface, more integrative functions and web-based tools. On the other hand, we will continue to collect more sequencing data to store in PIRD. In fact, we have sequenced and processed more than ten thousand samples that will be deposited in PIRD in the near future. Additionally, we will continue to update the TBAdb data in real time. Currently, most sequences in TBAdb are TCR $\alpha \beta$ sequences, and since BCR-related studies are increasing, more BCR sequences associated with various antigens and diseases will also be included in future versions.

\section{CONCLUSIONS}

We have developed a database PIRD to collect and store the annotated TCR and BCR sequencing data. It is a multifunctional database, providing data storage and online analysis platform. Other investigators can reuse and compare the data in PIRD.

\section{ACKNOWLEDGEMENT}

This project was funded by the Beijing Genomics Institute and China National GeneBank. The funder provided support in the form of salaries of all authors.

\section{FUNDING}

This work was supported in part by Shenzhen Municipal Government of China (JCYJ20160510141910129), and by the Science, Technology and Innovation Commission of Shenzhen Municipality under No. JCYJ20170817145404433 and JCYJ20170817145428361.

\section{CONFLICT OF INTEREST}

The authors declare that they have no competing interests. 


\section{REFERENCES}

Altschul, S.F., et al. (1990) Basic local alignment search tool, Journal of molecular biology, 215, 403-410.

Bolotin, D.A., et al. (2015) MiXCR: software for comprehensive adaptive immunity profiling, Nature methods, 12, 380-381.

Bolotin, D.A., et al. (2013) MiTCR: software for T-cell receptor sequencing data analysis, Nature methods, 10, 813-814.

Boyd, S.D., et al. (2009) Measurement and clinical monitoring of human lymphocyte clonality by massively parallel VDJ pyrosequencing, Science translational medicine, $\mathbf{1}$, 12 ra23.

Cheung, W.C., et al. (2012) A proteomics approach for the identification and cloning of monoclonal antibodies from serum, Nature biotechnology, 30, 447-452.

Christley, S., et al. (2018) VDJServer: A Cloud-Based Analysis Portal and Data Commons for Immune Repertoire Sequences and Rearrangements, Frontiers in immunology, 9, 976.

Corrie, B.D., et al. (2018) iReceptor: A platform for querying and analyzing antibody/B-cell and T-cell receptor repertoire data across federated repositories, Immunological reviews,

284, 24-41.

Dash, P., et al. (2017) Quantifiable predictive features define epitope-specific T cell receptor repertoires, Nature, 547, 89-93.

DeKosky, B.J., et al. (2013) High-throughput sequencing of the paired human

immunoglobulin heavy and light chain repertoire, Nature biotechnology, 31, 166-169.

DeKosky, B.J., et al. (2015) In-depth determination and analysis of the human paired heavyand light-chain antibody repertoire, Nature medicine, 21, 86-91.

Emerson, R.O., et al. (2017) Immunosequencing identifies signatures of cytomegalovirus exposure history and HLA-mediated effects on the T cell repertoire, Nature genetics, 49, 659-665.

Georgiou, G., et al. (2014) The promise and challenge of high-throughput sequencing of the antibody repertoire, Nature biotechnology, 32, 158-168.

Glanville, J., et al. (2017) Identifying specificity groups in the T cell receptor repertoire, Nature, 547, 94-98.

Glanville, J., et al. (2011) Naive antibody gene-segment frequencies are heritable and unaltered by chronic lymphocyte ablation, Proceedings of the National Academy of Sciences of the United States of America, 108, 20066-20071.

Gomez-Tourino, I., et al. (2017) T cell receptor beta-chains display abnormal shortening and repertoire sharing in type 1 diabetes, Nature communications, 8, 1792.

Gupta, N.T., et al. (2015) Change-O: a toolkit for analyzing large-scale B cell immunoglobulin repertoire sequencing data, Bioinformatics, 31, 3356-3358.

Howie, B., et al. (2015) High-throughput pairing of T cell receptor alpha and beta sequences, Science translational medicine, 7, 301ra131.

Huang, C., et al. (2019) The landscape and diagnostic potential of T and B cell repertoire in Immunoglobulin A Nephropathy, Journal of autoimmunity, 97, 100-107.

Huang, J., et al. (2017) A reference human genome dataset of the BGISEQ-500 sequencer, GigaScience, 6, 1-9.

Jackson, K.J., et al. (2014) Human responses to influenza vaccination show seroconversion signatures and convergent antibody rearrangements, Cell host \& microbe, 16, 105-114. 
Jardine, J.G., et al. (2016) HIV-1 broadly neutralizing antibody precursor B cells revealed by germline-targeting immunogen, Science, 351, 1458-1463.

Jiang, N., et al. (2013) Lineage structure of the human antibody repertoire in response to influenza vaccination, Science translational medicine, 5, 171ra119.

Khan, T.A., et al. (2016) Accurate and predictive antibody repertoire profiling by molecular amplification fingerprinting, Science advances, 2, e1501371.

Liu, X. and Wu, J. (2018) History, applications, and challenges of immune repertoire research, Cell biology and toxicology.

Liu, X., et al. (2016) Systematic Comparative Evaluation of Methods for Investigating the TCRbeta Repertoire, PloS one, 11, e0152464.

Parameswaran, P., et al. (2013) Convergent antibody signatures in human dengue, Cell host \& microbe, $13,691-700$.

Reuben, A., et al. (2017) TCR Repertoire Intratumor Heterogeneity in Localized Lung

Adenocarcinomas: An Association with Predicted Neoantigen Heterogeneity and

Postsurgical Recurrence, Cancer discovery, 7, 1088-1097.

Riaz, N., et al. (2017) Tumor and Microenvironment Evolution during Immunotherapy with Nivolumab, Cell, 171, 934-949 e915.

Robins, H.S., et al. (2009) Comprehensive assessment of T-cell receptor beta-chain diversity in alphabeta T cells, Blood, 114, 4099-4107.

Rosenfeld, A.M., et al. (2017) ImmuneDB: a system for the analysis and exploration of highthroughput adaptive immune receptor sequencing data, Bioinformatics, 33, 292-293.

Rosenfeld, A.M., et al. (2018) ImmuneDB, a Novel Tool for the Analysis, Storage, and Dissemination of Immune Repertoire Sequencing Data, Frontiers in immunology, 9, 2107. Rubelt, F., et al. (2017) Adaptive Immune Receptor Repertoire Community recommendations for sharing immune-repertoire sequencing data, Nature immunology, 18, 1274-1278.

Shugay, M., et al. (2018) VDJdb: a curated database of T-cell receptor sequences with known antigen specificity, Nucleic acids research, 46, D419-D427.

Smithey, M.J., et al. (2018) Lifelong CMV infection improves immune defense in old mice by broadening the mobilized TCR repertoire against third-party infection, Proceedings of the National Academy of Sciences of the United States of America, 115, E6817-E6825.

Tickotsky, N., et al. (2017) McPAS-TCR: a manually curated catalogue of pathologyassociated T cell receptor sequences, Bioinformatics, 33, 2924-2929.

Turchaninova, M.A., et al. (2016) High-quality full-length immunoglobulin profiling with unique molecular barcoding, Nature protocols, 11, 1599-1616.

Venturi, V., et al. (2008) The molecular basis for public T-cell responses?, Nature reviews. Immunology, 8, 231-238.

Vollmers, C., et al. (2013) Genetic measurement of memory B-cell recall using antibody repertoire sequencing, Proceedings of the National Academy of Sciences of the United States of America, 110, 13463-13468.

Wang, C., et al. (2015) B-cell repertoire responses to varicella-zoster vaccination in human identical twins, Proceedings of the National Academy of Sciences of the United States of America, 112, 500-505.

Wang, L., et al. (2018) Corrigendum: A Comprehensive Analysis of the T and B Lymphocytes Repertoire Shaped by HIV Vaccines, Frontiers in immunology, 9, 2931.

Wang, T., et al. (2017) The Different T-cell Receptor Repertoires in Breast Cancer Tumors, Draining Lymph Nodes, and Adjacent Tissues, Cancer immunology research, 5, 148-156. 
Warren, R.L., et al. (2011) Exhaustive T-cell repertoire sequencing of human peripheral blood samples reveals signatures of antigen selection and a directly measured repertoire size of at least 1 million clonotypes, Genome research, 21, 790-797. Weinstein, J.A., et al. (2009) High-throughput sequencing of the zebrafish antibody repertoire, Science, 324, 807-810.

Wu, D., et al. (2012) High-throughput sequencing detects minimal residual disease in acute T lymphoblastic leukemia, Science translational medicine, 4, 134 ra163.

Wu, J., et al. (2016) Minimal Residual Disease Detection and Evolved IGH Clones Analysis in Acute B Lymphoblastic Leukemia Using IGH Deep Sequencing, Frontiers in immunology, 7, 403.

Xiao Liu, W.Z., Jinghua Wu, Liya Lin, Jianzheng Nie, Ming Zhao and Qianjin Lu (2018) PanImmunome Initiative: Building an integrated $T$ and $B$ cell receptor repertoire map for immune-related diseases, The Journal of Immunology, 45.35.

Ye, J., et al. (2013) IgBLAST: an immunoglobulin variable domain sequence analysis tool, Nucleic acids research, 41, W34-40.

Ye, J., McGinnis, S. and Madden, T.L. (2006) BLAST: improvements for better sequence analysis, Nucleic acids research, 34, W6-9.

Zhang, W., et al. (2015) IMonitor: A Robust Pipeline for TCR and BCR Repertoire Analysis, Genetics, 201, 459-472.

Zhang, W., et al. (2017) Characterization of the B Cell Receptor Repertoire in the Intestinal Mucosa and of Tumor-Infiltrating Lymphocytes in Colorectal Adenoma and Carcinoma, Journal of immunology, 198, 3719-3728.

Zheng, C., et al. (2017) Landscape of Infiltrating T Cells in Liver Cancer Revealed by SingleCell Sequencing, Cell, 169, 1342-1356 e1316. 


\section{FIGURES and FIGURE LEGENDS}

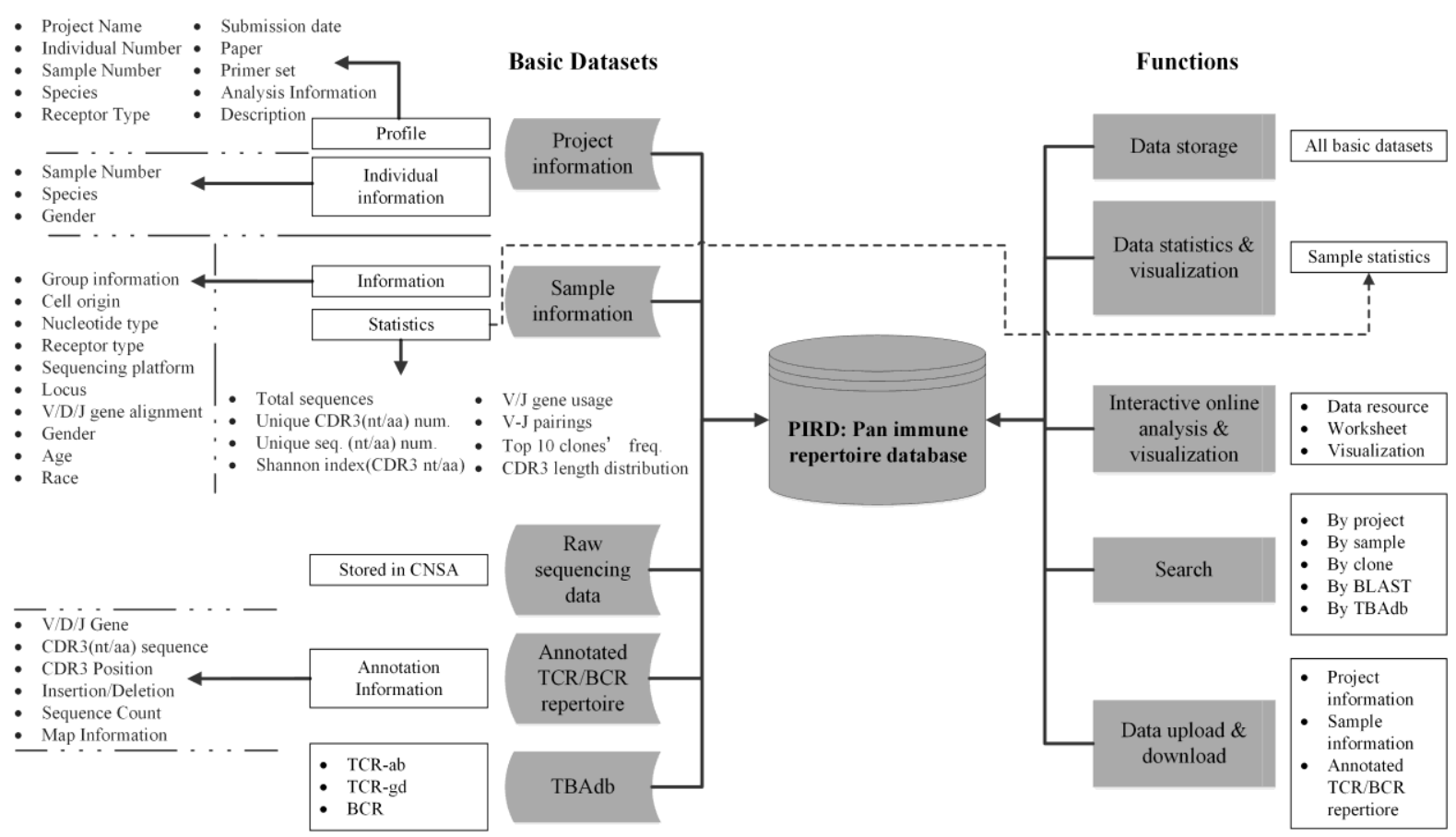

Figure 1. Systematic design of the PIRD database. In this database, there are five basic datasets and five functions. Each dataset or function includes one or multiple parts, and most parts contain several items. Partial items are listed on the left.

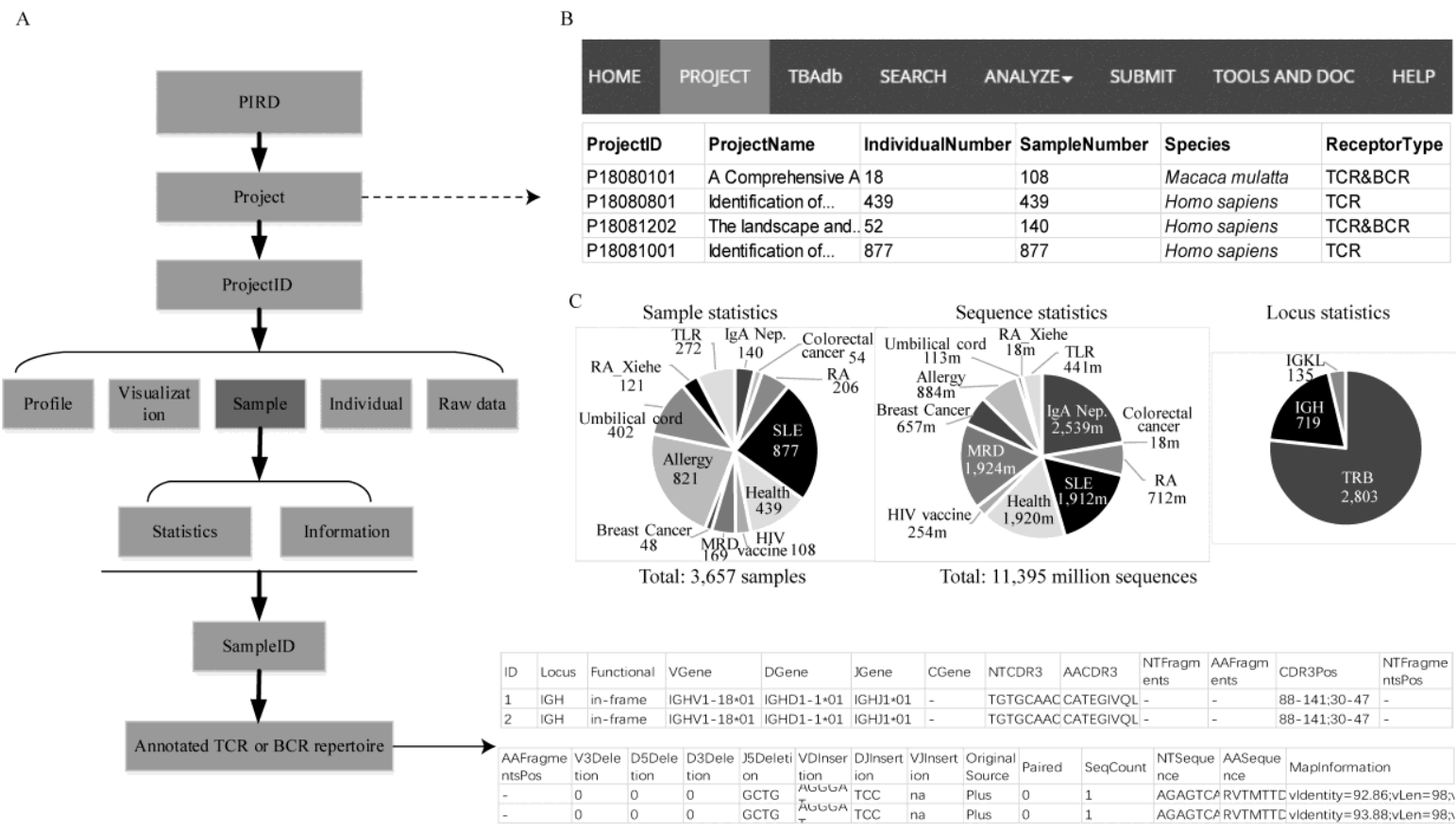

Figure 2. Detailed data deposited in PIRD. (A). Data storage structure for each project. By clicking the links of the PIRD website according to this structure, both sample-associated information and annotated TCR or BCR repertoire can be found. The right bottom corner displays 
an example of annotated BCR sequences. (B). Project page in PIRD. Partial projects are displayed here. (C). Data statistics in the current database (July 2019). m, million.

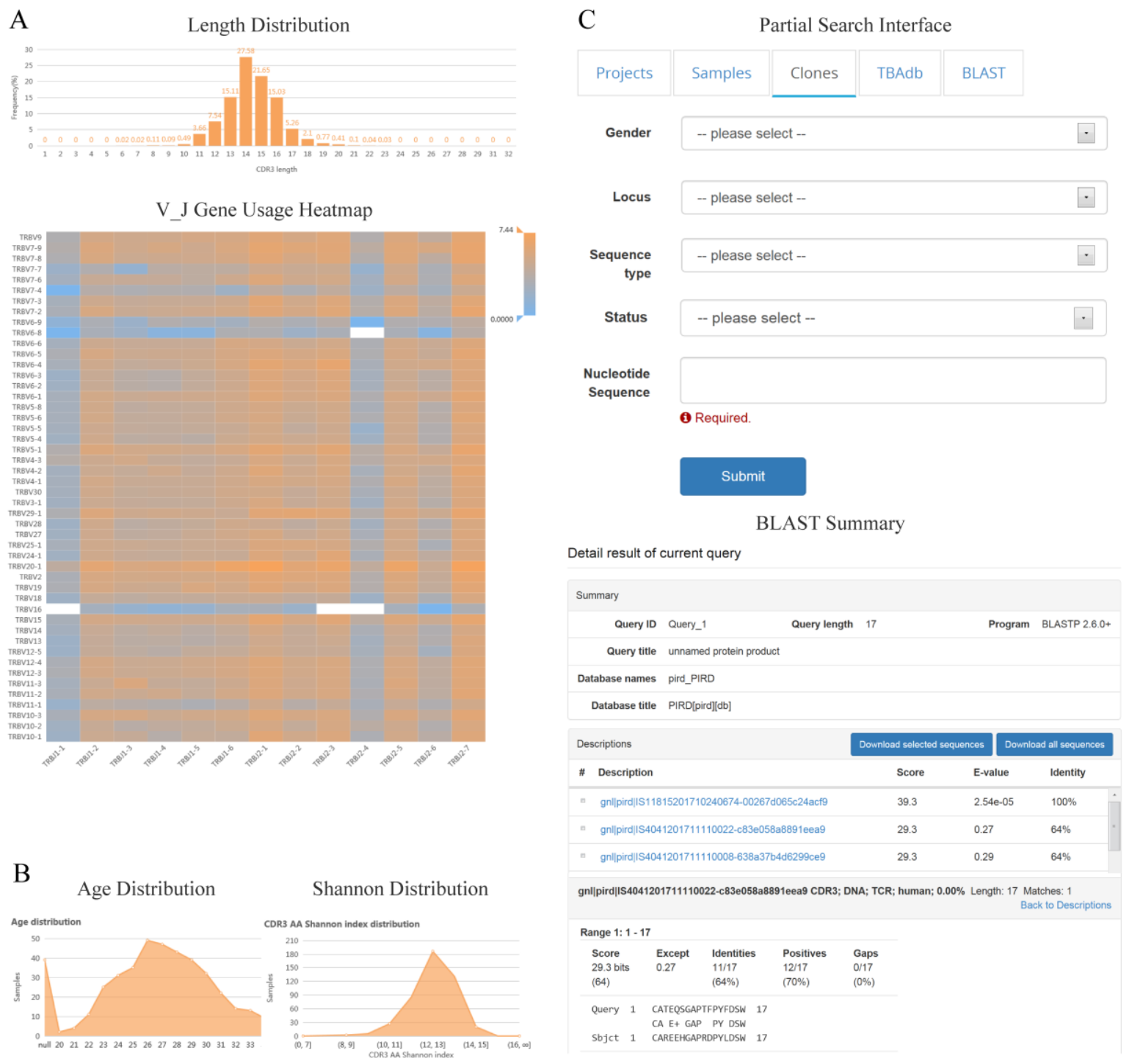

Figure 3. Examples of visualisation and search functions. (A). Visualisation of sample statistics. The length distribution of the CDR3 amino acid sequence (top panel) and the proportion of V-J gene pairings are represented by colour (bottom panel). (B). Visualisation of project information. The left panel shows the age distribution of samples, and the right panel shows the CDR3 diversity distribution in a project. (C). Search interface (top panel) and an example of local alignment search (bottom panel). The data in PIRD can be searched by project, sample, clone and TBAdb, and each provides multiple items to restrict the range of content. The sequences analogous to the query sequences can be found by the local alignment search where the tool BLAST is used. 


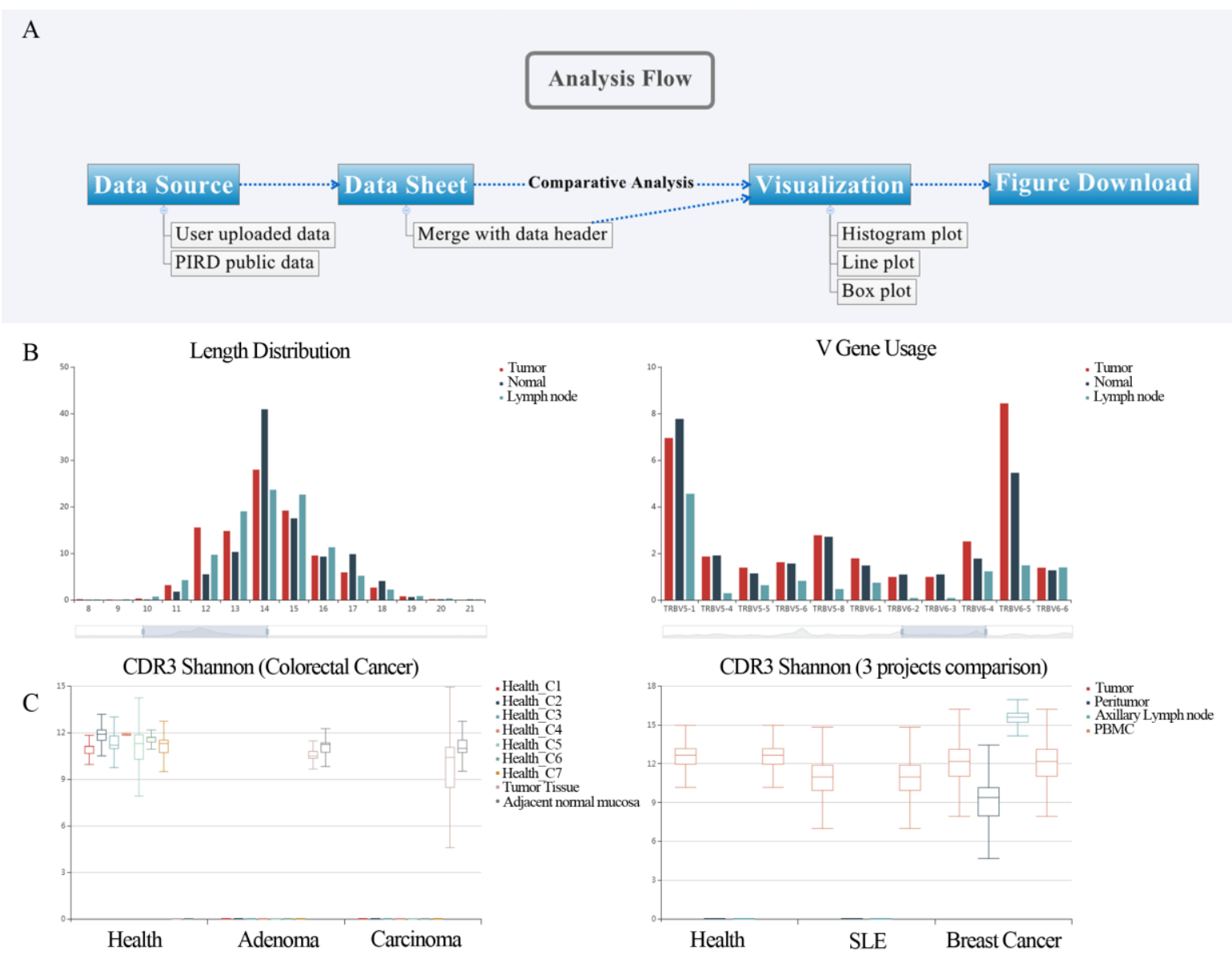

Figure 4. Examples of online interactive analysis and visualisation. (A). Flowchart of the analysis platform. (B). The length distribution of CDR3 amino acid sequences and TCR V $\beta$ gene usage from one patient (I190114010005) with breast cancer (P19011401). There are three samples from the patient, including tumour, adjacent normal tissue and lymph node. All TCR V $\beta$ gene usage can be viewed by sliding the lower box on the website. (C) The diversity of CDR3 amino acid sequences for different groups. The left panel shows the BCR diversities of intestinal samples derived from health, adenoma and carcinoma (P18081201). Each healthy individual contains seven intestinal segments $(\mathrm{C} 1, \mathrm{C} 2, \mathrm{C} 3, \mathrm{C} 4, \mathrm{C} 5, \mathrm{C} 6$ and $\mathrm{C} 7)$. The right panel shows the TCR repertoire's diversities of three projects, including health (P18080801), SLE (P18081001) and breast cancer (P19011401). P19011401 contains samples from tumour, adjacent normal tissue, lymph node and peripheral blood mononuclear cells (PBMC). The diversity is represented by the Shannon index. All data were selected from PIRD. All results of $(B)$ and $(C)$ were obtained using the analysis platform in PIRD. 
A

$\mathrm{D}$

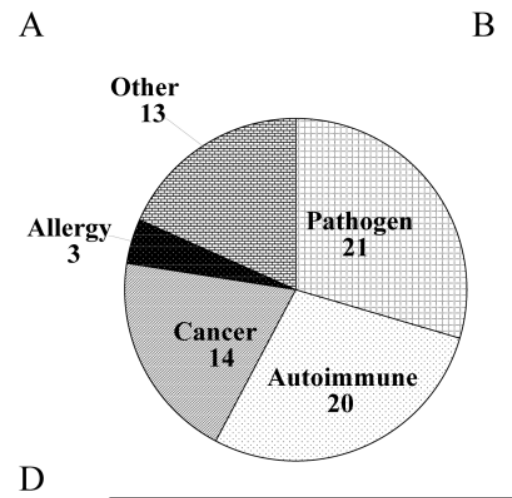

B

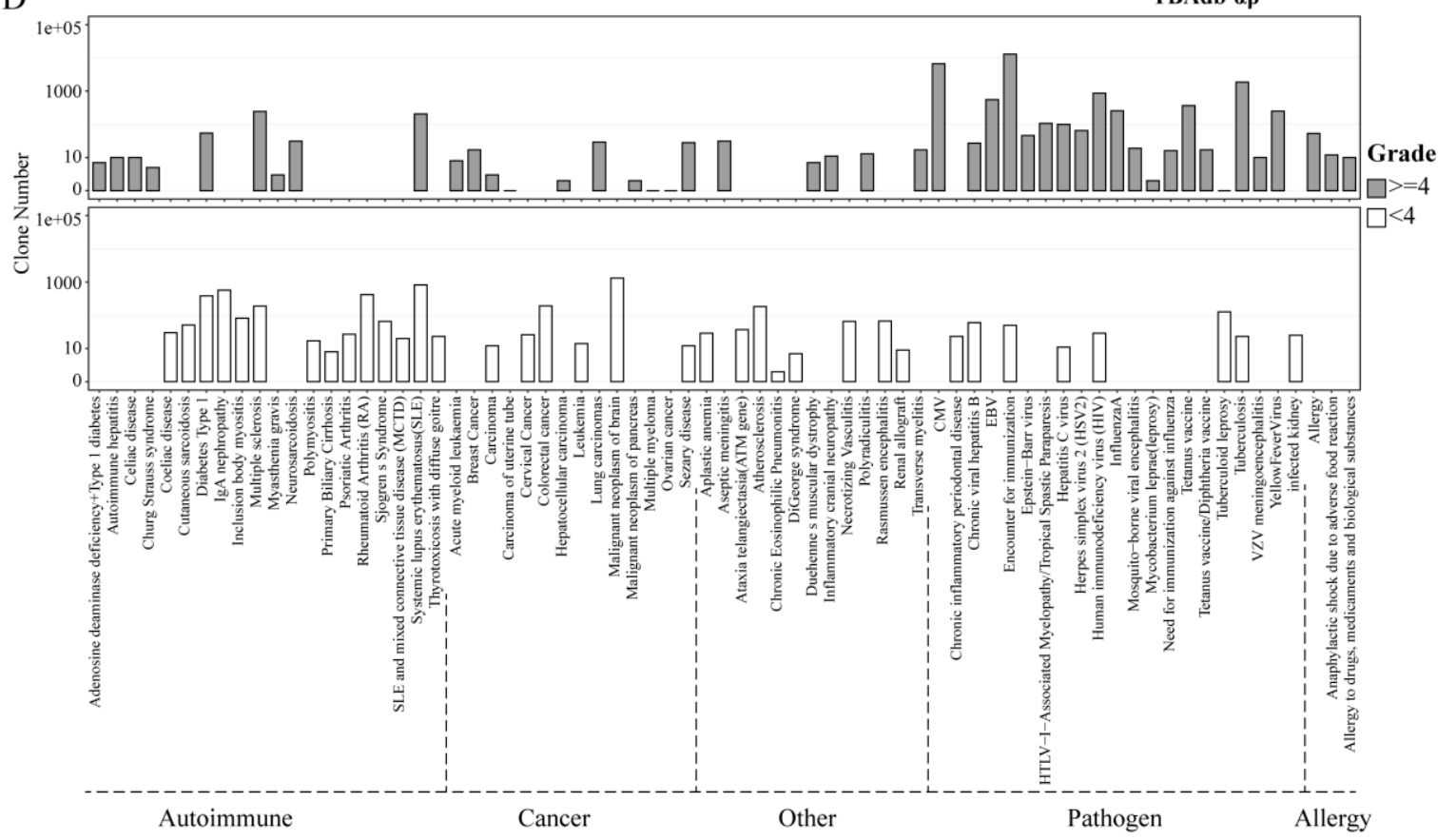

Figure 5. Overall summary of antigen-associated sequences in TBAdb. (A). The number of diseases in five disease categories. (B). The proportion of sequences in each disease category. (C). Comparison of TCR $\alpha \beta$ sequences in TBAdb (TBAdb-ab) with VDJdb and McPAS-TCR. V, VDJdb; M, McPAS-TCR; T, TBAdb. (D). The distribution of sequence amount for each disease in TBAdb. The upper panel shows the sequences with grade greater than 4 , and the bottom panel shows the sequences with grade less than 4 . The grade is a score to evaluate the reliability of sequences associated with the antigen. 'Other' signifies diseases that could not be classified into any of the other four disease categories. 